\title{
MCF-7 breast cancer cell line PacBio generated transcriptome has 300 novel transcribed regions, un-annotated in both RefSeq and GENCODE, and absent in the liver, heart and brain transcriptomes Sandeep Chakraborty, R - 44/ 1, Celia Engineers, T. T. C Industrial Area, Rabale, Navi Mumbai, 400701, India.
}

\begin{abstract}
Illuminating the 'dark' regions of the human genome remains an ongoing effort, a decade and a half after the human genome was sequenced - RefSeq and GENCODE being two of the major annotation databases. Pacific Biosciences (PacBio) has provided open access to the transcriptome of MCF-7, a breast cancer cell line that has provided significant therapeutic advancement in breast cancer research since the 1970s. PacBio sequencing generates much longer reads compared to second-generation sequencing technologies, with a trade-off of lower throughput, higher error rate and more cost per base. Here, this transcriptome was analyzed using the YeATS pipeline, with additionally introduced kmer based algorithms, reducing computational times to a few hours on a simple workstation. Out of $\sim 300$ transcripts that have no match in both RefSeq and GENCODE, $\sim 250$ are absent in the transcriptomes of the heart, liver and brain, also provided by PacBio. Also, $\sim 200$ transcripts are absent in a recent catalogue of un-annotated long non-coding RNAs from 6,503 samples ( 43 Terabases of sequence data) [1], and among 2,556 novel transcripts reported in an experimental workflow RACE-Seq [2]. 65 transcripts have $>100$ amino acid open reading frames, and have the potential of being protein coding genes. ORF based annotation also identified few bacterial transcripts in the PacBio database mapped to the human genome, and one human transcript that has been annotated as bacterial in the NCBI database. The current work reiterates the under-utilization of transcriptomes for annotating genomes. It also provides new leads for investigating breast cancer by virtue of exclusively expressed transcripts not expressed in other tissues, which have the prospects of breast cancer biomarkers based on further investigations.
\end{abstract}




\section{Introduction}

The initial estimates of 25,000 protein coding genes in humans has been moderated to about 19,000 recently [3]. This constitutes $\sim 1 \%$ of the genome, but most of the $99 \%$ 'dark' genome plays significant regulatory roles in the cellular machinery [4]. The annotation of these regions is critical for correlating disease to genomic variants [5]. The two major independent annotation databases, periodically updated, are RefSeq [6] and GENCODE [7].

Pacific Biosciences (PacBio) sequencing [8] generates much longer reads compared to second-generation sequencing technologies [9], with a trade-off of lower throughput, higher error rate and more cost per base [10, 11]. The longer sequence lengths in PacBio compared to other sequencing methods might alleviate assembly issues associated with other methods with shorter read lengths $[12,13]$. PacBio has provided open access to the transcriptome of the MCF-7 breast cancer cell line [14,15]. There are currently two versions - one provided in 2013 and one in 2015. Two novel long non-coding RNAs were discovered in human mitochondrial DNA using the 2015 database by a different group [16].

The under-utilization of transcriptomes while annotating genomes [17-19] was recently emphasized for the walnut genome [20]. Here, the publicly available transcriptome of the MCF-7 breast cancer cell line (2013 version) was used to find novel human transcripts that are not annotated in current databases. $\sim 300$ transcripts are identified in the MCF-7 cells that have no annotation in the current RefSeq and GENCODE databases. Moreover, most of these transcripts are absent in heart, liver and brain transcriptomes also provided by PacBio. Also, 200 transcripts are absent in a recent catalogue of un-annotated long noncoding RNAs (lncRNA) from 6,503 samples ( 43 Terabases of sequence data) [1]. Furthermore, there no common transcripts with another experimental workflow RACE-Seq (rapid amplification of cDNA ends) and long-read RNA sequencing that reported 2,556 novel transcripts [2]. 65 of these transcripts have $>100$ long open reading frames (ORF), and might represent novel protein coding genes. A criteria comparing the amino acid frequency of the ORFs to the standard amino acid frequency found in human proteins is described in order to exclude proteins enriched for single amino acids which have repetitive sequences. Also, comparison of the ORFs to the BLAST 'nr' database highlights certain bacterial transcipts (Accid:WP_069187498.1) mapped to the human genome in the PacBio dataset, and one human protein (Accid:CPR56970.1) being annotated as a Chlamydia trachomatis protein in the NCBI database.

\section{Materials and methods}

\section{GENCODE dataset}

GENCODE release 25 was downloaded from https://www.gencodegenes.org/ (release date 07/2016). Two files - gencode.v25.transcripts.fa $(n=200 \mathrm{k})$ and gencode.v25.lncRNA_transcripts.fa $(\mathrm{n}=27 \mathrm{k})$ - were combined to create a single database(GENCODE.NTDB).

\section{RefSeq dataset}

The RefSeq database was created from https://www.ncbi.nlm.nih.gov/nuccore choosing mRNA, rRNA, cRNA, tRNA and ncRNA sequences (FILE:mrna.refseq.160k.fa, n=161k, REFSEQ.NTDB).

\section{PacBio dataset}

The MCF-7 transcriptome was obtained from http://www.pacb.com/blog/data-release-human-mcf-7-transcriptome (2013 version). There is another updated version made available in 2015, that has not been analyzed in this study. The PacBio dataset for human heart, liver and brain transcriptomes is available at http://datasets.pacb.com.s3.amazonaws.com/2014/Iso-seq_Human_Tissues/list.html and provides 'a dataset containing the full-length whole transcriptome from three diverse human tissues (brain, heart, and liver). The updated version of the Iso-Seq method incorporates the use of a new PCR polymerase that improves the 
representation of larger transcripts, enabling sequencing of cDNAs of nearly $10 \mathrm{~kb}$ in length. The inclusion of multiple sample types makes this dataset ideal for exploring differential alternative splicing events' [21]. The transcripts from all three tissues were concatenated to create a single file (FILE:PacBioHLB.fa, n=23309). The transcripts have been renamed to allow Unix style filenames.

\section{kmer analysis}

The MCF-7 transcriptome was 30kmer compared to the GENCODE.NTDB and REFSEQ.NTDB. This step removes all transcripts that have at least a 30 long sequence (ignoring repetitive sequences) common in the respective annotation databases. This is a conservative step that just reduces the search database, since a transcript ignored as mentioned above might still not match exactly to any entry in the annotation database. The 'unmatched' transcipts are now BLAST'ed [22] to GENCODE.NTDB and REFSEQ.NTDB to have a complete alignment. This results in two sets of transcripts. The first set has no matches in the database, and are definitely not annotated. The second set has homologous entries in the database, but need to be manually checked for homology. It is seen that entries which are $<96 \%$ homologous are mostly novel annotations. Open reading frames were obtained 'getorf' program from the EMBOSS suite [23]. Computational runtimes are very modest, and takes a few hours on a personal workstation.

\section{Results and discussion}

\section{No match in the RefSeq and GENCODE databases:}

First, transcripts that shared a 30kmer sequence with any annotated sequence in the GENCODE and RefSeq databases were removed. Next, a BLAST comparison of transcripts that do not share a $30 \mathrm{kmer}$ sequence in the respective GENCODE and RefSeq databases provided transcripts which have no matches in the GENCODE.NTDB (FILE:notinGENCODE.list, n=346) and REFSEQ.NTDB (FILE:notinREFSEQ.list, $\mathrm{n}=418$ ). This corroborates a recent study that found GENCODE to have better coverage [24]. There are 252 transcipts common to these sets.

\section{Partial homology in the RefSeq and GENCODE databases:}

The BLAST comparison also provided a list of transcripts with partial homology to the RefSeq and GENCODE databases. Transcripts with $>96 \%$ homology are probably annotated, and excluded. There are 42 transcripts that have $<96 \%$ homology with respect to both databases, and thus can be considered as not being annotated (FILE:notInBoth.96homology.list). These transcripts require manual inspection. 


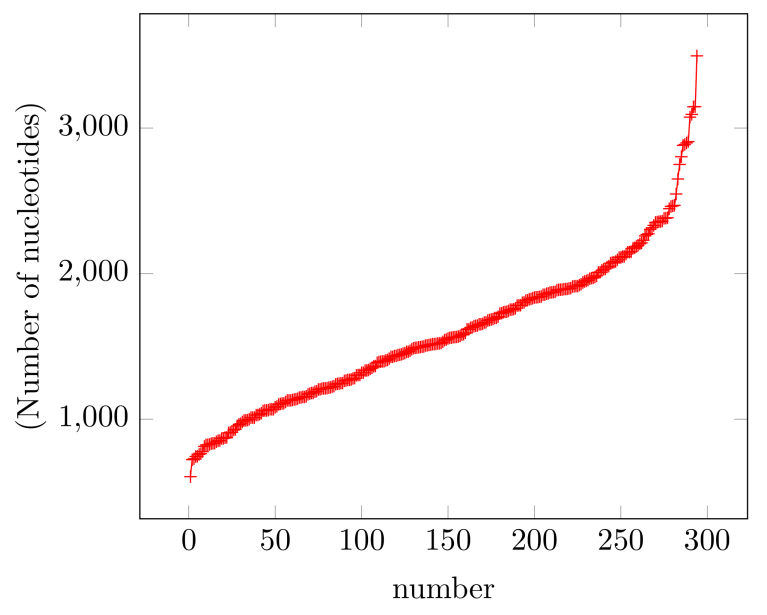

Figure 1: Length of the 294 transcripts not found in RefSeq and GENCODE databases: Shortest is $\sim 600$ nt long, while the longest is $\sim 3500 \mathrm{nt}$. In contrast the mitranscriptome.org database has $\sim 12 \mathrm{k}$ sequences ranging from 300 to $985680 \mathrm{nt}$.

\section{Comparison to the PacBio transcriptome of the heart, liver and brain}

Next, these transcripts were combined (FILE:notInBoth.list, n=294, Fig 1) and compared to the PacBio provided dataset containing the full-length whole transcriptome from three diverse human tissues (brain, heart, and liver, FILE:PacBioHLB.fa). There were 264 transcripts that were not present in these tissues (FILE:notin.HLBdatabase.list), thus representing transcripts exclusive to the MCF-7 cell line (at least with respect to the brain, heart, and liver).

\section{Comparison to the mitranscriptome.org database}

The relevance of lncRNAs in cancer studies has long been established [25]. A recent work classified 58,648 genes as lncRNAs after a comprehensive analysis of '7,256 RNA-Seq libraries from tumors, normal tissues, and cell lines comprising over 43 terabases of sequence from 25 independent studies' [1]. Supplementary Table 10 (http://www.nature.com/ng/journal/v47/n3/full/ng.3192.html\#supplementary-information) provides the genomic coordinates of these lncRNAs, which was extracted using 'http://genome.ucsc.edu/cgibin/das/hg19/dna?segment=CHR:start,end'. This information is also provided in http://mitranscriptome.org/. Follow-up work on this database demonstrated the importance of specific lncRNAs to breast cancer [26]. There are $\sim 12 \mathrm{k}$ sequences ranging from 300 to 985680 nucleotides. Comparison of the 294 transcripts (FILE:notInBoth.list) to these sequences identified common transcripts (FILE:mitranscriptome.same, $\mathrm{n}=52$ ) and unannotated transcripts (FILE:mitranscriptome.NOTTHERE, $\mathrm{n}=242$ ). Unannotated transcripts include those that have homology (> BLAST bitscore 100) but map to a different chromosome, as well as those that have no homologs. 
Table 1: Transcripts with ORFs $>\mathbf{1 0 0}$ aa long that match to the 'nr' database: Transcript 1 , 2 and 4 are bacterial transcripts, demonstrating the necessity of removing metagenomic transcripts prior to analysis. Transcript 8 is misannotated as protein from Chlamydia trachomatis. The transcript (Accid:CSTN01000036.1) linked to the protein (Accid:CPR56970.1) matches to the human genome, and not to Chlamydia trachomatis. A cutoff of BLAST bit score (BBS) of 100 was used. The other $\sim 90$ transcripts might represent novel protein coding genes or lncRNAs.

\begin{tabular}{|c|c|c|c|c|}
\hline Idx & Transcript & Accid & Description & BBS \\
\hline 1 & CHR16.46389879_46398888_I3_C30162.F2P21.3075.RT_29 & WP_069187498.1 & [S. pneumoniae] & 247 \\
2 & CHR16.46386138_46388586_.I2A_C135052.F1P32.2469.RT_26 & CJE27995.1 & [S. pneumoniae] & 216 \\
3 & CHR19.39155468_39158343+.I2A_C37015.F3P20.2881.RT_18 & EAW56812.1 & [H. sapiens] & 197 \\
4 & CHR16.46386138_46388586_.I2A_C135052.F1P32.2469.RT_36 & CKT10751.1 & {$[$ M. tuberculosis] } & 178 \\
5 & CHR15.99344096_99385537+.I1D_C21966.F4P10.1565.RT_15 & EAX02227.1 & [H. sapiens] & 140 \\
6 & CHR11.70996950_70998864+.I1C_C72675.F1P38.1921.RT_32 & AIE45854.1 & [synthetic construct] & 119 \\
7 & CHR2.12841430_12856867_.I1D_C30377.F5P15.1894.RT_31 & XP_011747760.1 & [M. nemestrina] & 112 \\
8 & CHR1.145370688_145372138+.I1A_C27600.F1P13.1454.RT_5 & CPR56970.1 & [C. trachomatis] & 105 \\
9 & CHR7.50518150_50519652+.I1D_C31040.F3P6.1510.RT_2 & EAW60974.1 & [H. sapiens] & 101 \\
\hline
\end{tabular}

\section{Comparison to the RACE-seq novel transcripts:}

Another experimental workflow RACE-Seq (rapid amplification of cDNA ends) and long-read RNA sequencing reported 2,556 novel transcripts [2]. The genomic coordinates are obtained from https://public_docs.crg.es/rguigo/Papers/ uszczynska_RACE-Seq/ (FILE:phase6-clean.bed, $n=2486$ ). None of the novel transcripts identified here were reported in this study (FILE:RACESEQ.THERE).

\section{Identification of possible protein coding genes:}

Transcripts encoding open reading frames (ORF) longer than 100 amino acids are not typically considered as lncRNAs [27]. There are 65 transcripts with $>100$ long ORFs (FILE:notInBoth.BRCA.list.ORF.100.fa). The amino acid frequency (AAF) of these ORFs can be compared to the combined AAFs found in all human proteins (Fig 2) to select more likely protein coding genes.

\section{Misannotated transcripts in the PacBio dataset and NCBI dataset}

These ORFs were BLAST'ed to the complete 'nr' database (Table 1). This highlighted that bacterial transcipts from Streptococcus pneumoniae and Mycobacterium tuberculosis have being annotated as human transcipts in the PacBio database. For example, CHR16.46389879_46398888_.I3_C30162.F2P21.3075.RT has a $89 \%$ homology to chr16 (which explains the annotation), and no homology to S. pneumoniae. However, it encodes an ORF (132 aa long) which has a 100\% identity to a protein from S. pneumoniae (Accid:WP_069187498.1). This emphasizes the importance of using ORF based annotation for transcripts with low homology [28]. Another human protein (Accid:CPR56970.1) has been mistakenly annotated as a Chlamydia trachomatis protein in the NCBI database, based on a vaginal swab transcript (Accid:CSTN01000036.1, $2920 \mathrm{nt}$ ) that has a $99 \%$ homology to the human chromosome 1.

\section{Conclusions:}

The current work highlights the 'low hanging fruits' still available for widely researched diseases like breast cancer, at very low computational costs. The technological advancement provided by PacBio sequencing might be responsible for identification of these transcripts, that has eluded detection thus far. It also highlights the necessity of metagenomic filtration prior to analysis. 


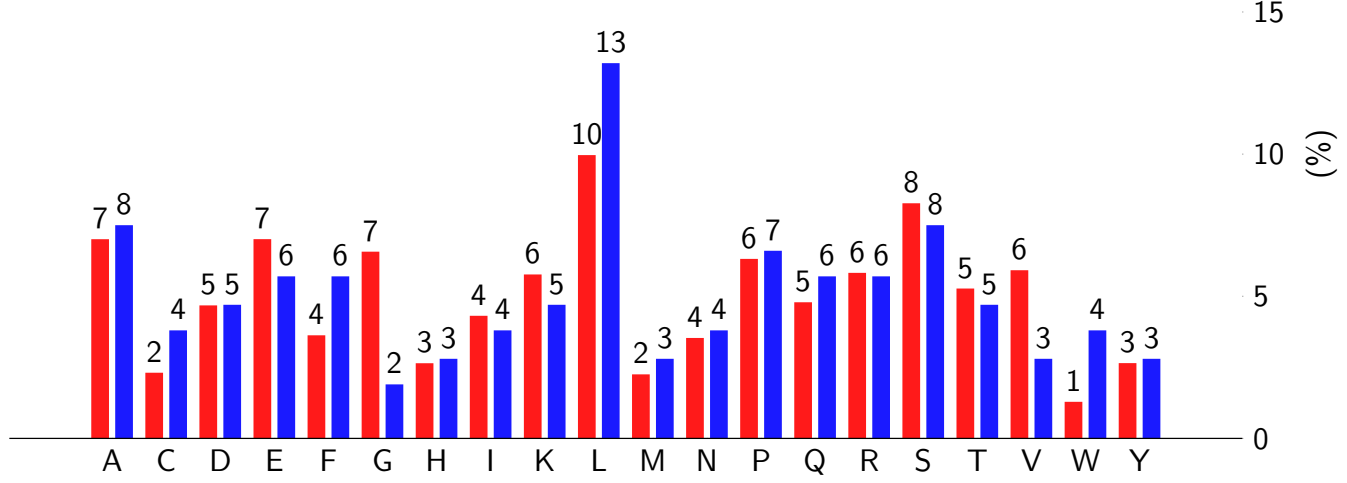

Figure 2: Amino acid frequency (AAF) in human proteins: The cumulative AAF (in red) is obtained from the ncbi RefSeq human proteins $(n=80 \mathrm{k})$. The AAF for the ORF from the transcript CHR5.67101977_67106707+.I3_C6784.F3P34.3147.RT_6 (length=106 aa) is in blue.

\section{Revisions from version 1:}

1. The number of transcripts having ORFs $>100$ aa is updated to 65 from $\sim 100$. The program for analyzing was considering mutliple ORFs from the same transcript previously - this has been fixed.

2. It was previously mentioned that there were two transcripts that were common in the $\sim 2500$ novel transcripts identied in the RACE-seq study. The homology of these matches is too low to classify these as matches.

\section{References}

1. Iyer MK, Niknafs YS, Malik R, Singhal U, Sahu A, et al. (2015) The landscape of long noncoding rnas in the human transcriptome. Nature genetics 47: 199-208.

2. Lagarde J, Uszczynska-Ratajczak B, Santoyo-Lopez J, Gonzalez JM, Tapanari E, et al. (2016) Extension of human lncrna transcripts by race coupled with long-read high-throughput sequencing (race-seq). Nature communications 7.

3. Ezkurdia I, Juan D, Rodriguez JM, Frankish A, Diekhans M, et al. (2014) Multiple evidence strands suggest that there may be as few as 19000 human protein-coding genes. Human molecular genetics 23: $5866-5878$.

4. Chi KR (2016) The dark side of the human genome. Nature 538: 275-277.

5. Sjöblom T, Jones S, Wood LD, Parsons DW, Lin J, et al. (2006) The consensus coding sequences of human breast and colorectal cancers. science 314: 268-274.

6. Pruitt KD, Tatusova T, Maglott DR (2007) Ncbi reference sequences (refseq): a curated non-redundant sequence database of genomes, transcripts and proteins. Nucleic acids research 35: D61-D65. 
7. Harrow J, Frankish A, Gonzalez JM, Tapanari E, Diekhans M, et al. (2012) Gencode: the reference human genome annotation for the encode project. Genome research 22: 1760-1774.

8. Eid J, Fehr A, Gray J, Luong K, Lyle J, et al. (2009) Real-time dna sequencing from single polymerase molecules. Science 323: 133-138.

9. Quail MA, Smith M, Coupland P, Otto TD, Harris SR, et al. (2012) A tale of three next generation sequencing platforms: comparison of ion torrent, pacific biosciences and illumina miseq sequencers. BMC genomics 13: 1.

10. Rhoads A, Au KF (2015) Pacbio sequencing and its applications. Genomics, proteomics \& bioinformatics 13: 278-289.

11. English AC, Richards S, Han Y, Wang M, Vee V, et al. (2012) Mind the gap: upgrading genomes with pacific biosciences rs long-read sequencing technology. PloS one 7: e47768.

12. Chakraborty S (2016) Rna-seq assembler artifacts can bias expression counts and differential expression analysis - case study on the chickpea transcriptome emphasizes importance of freely accessible data for reproducibility [version 2; referees: 2 not approved]. F1000Research 5.

13. Steijger T, Abril JF, Engström PG, Kokocinski F, Hubbard TJ, et al. (2013) Assessment of transcript reconstruction methods for rna-seq. Nature methods 10: 1177-1184.

14. Soule H, Vazquez J, Long A, Albert S, Brennan M (1973) A human cell line from a pleural effusion derived from a breast carcinoma. Journal of the National Cancer Institute 51: 1409-1416.

15. Lee AV, Oesterreich S, Davidson NE (2015) Mcf-7 cellschanging the course of breast cancer research and care for 45 years. Journal of the National Cancer Institute 107: djv073.

16. Shan G, Tian X, Sun Y, Wu Z, Cheng Z, et al. (2016) Two novel lncrnas discovered in human mitochondrial dna using pacbio full-length transcriptome data. bioRxiv : 079517.

17. Chakraborty S, Britton M, Wegrzyn J, Butterfield T, Martinez-Garcia PJ, et al. (2015). YeATS-a tool suite for analyzing RNA-seq derived transcriptome identifies a highly transcribed putative extensin in heartwood/sapwood transition zone in black walnut.

18. Chakraborty S, Britton M, Martínez-García P, Dandekar AM (2016) Deep RNA-seq profile reveals biodiversity, plant-microbe interactions and a large family of NBS-LRR resistance genes in walnut (juglans regia) tissues. AMB Express 6: 1.

19. Chakraborty S, Martinez-Garcia PJ, Dandekar A (2016). YeATSAM analysis of the walnut and chickpea transcriptome reveals key genes undetected by current annotation tools [version 1; referees: 1 approved, 1 not approved].

20. Martínez-García PJ, Crepeau MW, Puiu D, Gonzalez-Ibeas D, Whalen J, et al. (2016) The walnut (juglans regia) genome sequence reveals diversity in genes coding for the biosynthesis of nonstructural polyphenols. The Plant Journal .

21. Clark TA (2015) Single molecule, real-time sequencing of full-length cdna transcripts uncovers novel alternatively spliced isoforms. In: Plant and Animal Genome XXIII Conference. Plant and Animal Genome.

22. Camacho C, Madden T, Ma N, Tao T, Agarwala R, et al. (2013) BLAST Command Line Applications User Manual .

23. Rice P, Longden I, Bleasby A (2000) EMBOSS: the European Molecular Biology Open Software Suite. Trends Genet 16: 276-277. 
24. Frankish A, Uszczynska B, Ritchie GR, Gonzalez JM, Pervouchine D, et al. (2015) Comparison of gencode and refseq gene annotation and the impact of reference geneset on variant effect prediction. BMC genomics 16: S2.

25. Prensner JR, Chinnaiyan AM (2011) The emergence of lncrnas in cancer biology. Cancer discovery 1: 391-407.

26. Niknafs YS, Han S, Ma T, Speers C, Zhang C, et al. (2016) The lncrna landscape of breast cancer reveals a role for dscam-as1 in breast cancer progression. Nature communications 7.

27. Wang B, Tseng E, Regulski M, Clark TA, Hon T, et al. (2016) Unveiling the complexity of the maize transcriptome by single-molecule long-read sequencing. Nature communications 7.

28. Chakraborty S (2016) Transcriptome from saffron (crocus sativus) plants in jammu and kashmir reveals abundant soybean mosaic virus transcripts and several putative pathogen bacterial and fungal genera. bioRxiv : 079186. 